\title{
A clarification of frequency effects in children's discrimination learning ${ }^{*} \uparrow$
}

\author{
ELIZABETH S. GHATALA \\ Weber State College, Ogden, Utah 84403 \\ and \\ JOEL R. LEVIN and LOIS A. MAKOID \\ University of Wisconsin, Madison, Wisconsin 53706
}

\begin{abstract}
Three experiments were conducted to assess the effects of certain stimulus variables on children's discrimination learning. In general, it was found that word frequency was negatively related to discrimination learning as long as the words were meaningful to Ss. Moreover, the relationship between word frequency and performance reversed in free-recall learning, as was expected. Equivalent relationships between frequency and learning were obtained with both verbal and pictorial materials. The implications of these results were discussed in the context of popular accounts of memorial representation.
\end{abstract}

The purpose of the present series of experiments was to determine the effects of certain stimulus variables on children's discrimination learning. The three dimensions on which materials were varied were "background frequency" (as inferred from norms such as those of Thorndike \& Lorge, 1944), meaningfulness (as defined by $\mathrm{Ss}^{\prime}$ semantic responses to items), and modality (verbal vs pictorial representations).

According to the tenets of frequency theory (Ekstrand, Wallace, \& Underwood, 1966), (1) discrimination learning is assumed to involve subjective frequency discriminations between "correct" and "incorrect" pair members. It has been assumed further that (2) the accrual of subjective frequency to items in a pair may be influenced by the background or preexperimental frequency of the items in a manner akin to Weber's law. Under the second assumption, discrimination of situational frequency differences should be easier for items low in background frequency than for those high in background frequency.

In earlier studies (Ghatala \& Levin, 1973, 1974; Ghatala, Levin, Wilder, 1973; Levin, Ghatala, \& DeRose, in press), we have invoked the background frequency

\footnotetext{
* This research was funded by the Wisconsin Research and Development Center for Cognitive Learning, supported in part as a research and development center by funds from the $\mathrm{N}$ ational Institute of Education. The opinions herein do not necessarily reflect the position or policy of the National Institute of Education and no official endorsement by the National Institute should be inferred. Center No. NE-C-00-3-0065. We are grateful to Benton J. Underwood and Larry Wilder for their inputs during the planning of the study, to Jerri Belliston for collecting the data in Experiment $I$, to Ann $E$. McCabe for assistance in constructing the materials for Experiments II and III, to the staffs and students of Grandview and T. O. Smith Elementary Schools in Ogden, Utah, Caswell and Rockwell Elementary Schools in Fort Atkinson, Wisconsin, and Yahara Elementary School in Stoughton. Wisconsin, and to Cathy Bussey for typing the paper.

tRequests for reprints should be addressed to Elizabeth S. Ghatala, Department of Psychology, Weber State College, Ogden, Utah 84403.
}

assumption to account for various phenomena in children's discrimination learning. The emphasis in the present research is on clarifying the role of background frequency as it operates in conjunction with other stimulus variables previously demonstrated (or presumed) to have an effect on discrimination learning.

\section{EXPERIMENT I}

One of the assumptions just mentioned was that discrimination learning should be better with materials of low background frequency than with those of high background frequency. However, tests of this prediction utilizing high- and low-frequency words have been equivocal. While some studies have found that low-frequency word pairs are learned significantly better than high-frequency pairs (e.g., Rowe \& Paivio, 1971b, Experiments I and IV; Underwood, Broder, \& Zimmerman, 1973), others have not (e.g., Ingison \& Ekstrand, 1970; Paivio \& Rowe, 1970; Rowe \& Paivio, 1971b, Experiments II and III). And, as Paivio (1971) has argued, even when such word frequency effects are found, they are generally not as potent as those produced by other stimulus variables-in particular, stimulus concreteness as defined by Paivio, Yuille, and Madigan's (1968) norms.

Recently, Ghatala and Levin (1974) presented evidence to suggest that the elusive effect of word frequency in discrimination learning might be due to the operation of another factor which may be regarded as "meaningfulness" (though not in the usual verbal learning sense, e.g., Underwood \& Schulz, 1960). In a frequency judgment task, it was found that subjective frequency differences between high-frequency words and low-frequency words for which Ss knew the meanings were in accordance with predictions stemming from Weber's law. In contrast, the lack of difference 
between high-frequency words and low-frequency words for which Ss did not know the meanings was not in accordance with Weber's law. This was true even though the "meaningful" and "nonmeaningful" low-frequency words were fairly comparable in terms of their average normative (Thorndike \& Lorge, 1944) frequencies, these being 8.2 and 6.0 occurrences per million, respectively.

The Ghatala and Levin results indicated that, within the context of a frequency judgment task, Weber's law holds for materials which are meaningful to Ss but not for materials which are not meaningful. This finding in turn suggests that meaningfulness (as defined here) may well be a crucial variable to control when investigating the effects of background frequency in discrimination learning. Indeed, a preliminary experiment provided suggestive evidence for this hypothesis. Independent groups of sixth-grade children learned verbal discrimination lists consisting of (a) pairs of high-frequency words, (b) pairs of low-frequency words whose meanings were presumed (on the basis of prior ratings) to be known to Ss, (c) pairs of low-frequency words whose meaning were unknown to $S$, and (d) pairs of nonsense words. The predictions based on the Ghatala and Levin (1974) results were that the list consisting of low-frequency meaningful words would be learned better than the high-frequency word list and that the list consisting of low-frequency nonmeaning ful words would be of the same or greater difficulty as the high-frequency list. The worst performance relative to the high-frequency word condition was expected from the nonsense condition, since it represented an even lower extreme of the meaningfulness dimension (due to the items' lesser orthographic and phonetic resemblance to real English words).

Analysis of learning scores indicated that, while the nonsense condition was significantly inferior to the high-frequency word condition, neither of the two low-frequency lists differed from the high-frequency list. The absence of the predicted superiority of the low-frequency, meaningful word condition to the high-frequency word condition prompted a further analysis in which $S s$ in the former condition were given a definitions test on the words contained in the discrimination list. On the test the Ss were required to pronounce each word (if $S$ failed in pronouncing, $E$ pronounced the word for him) and then define it. The results obtained from this procedure indicated that, while the items had uniformly high meaningfulness (as had been established by Ghatala \& Levin, 1974), individual $S s$ exhibited considerable variation in their ability to pronounce and define the words. Of particular interest was the significant $(p<.01)$ correlation between number of words correctly recognized (pronounced and defined) and total correct on the discrimination task: a trend which supports the original prediction in that, as Ss' semantic knowledge of the words increased, so did their discrimination learning scores.

Performance on the definitions test suggested that with certain procedural changes the relationship between frequency and meaningfulness in discrimination learning might be more clearly observed. In particular, it was noted that many Ss could provide a defirition of the words once they were pronounced for them. And the Ghatala and Levin (1974) results which led to the present predictions were based on instructions to Ss to read each item to themselves as it appeared-instructions which were not explicitly stated in the Procedure section of that paper. Based on this evidence, we decided to conduct the experiment with all items pronounced for Ss during learning.

\section{Method}

Subjects. The Ss were 48 sixth-grade children attending an elementary school located in a middle-class neighborhood in Ogden, Utah. The Ss were randomly assigned in equal numbers to the three conditions of the experiment. The nonsense condition of the preliminary study was eliminated here because it produced the predicted poorer performance under previous procedures and because it was not crucial to the basic hypothesis.

Design and Materials. One list (Hi-F) consisted of high-frequency concrete nouns from the AA and $A$ range of the Thorndike and Lorge (1944) norms. The second list (Lo-F/Hi-M) consisted of low-frequency concrete nouns (less than 25 occurrences per million) whose meanings were known by $\mathrm{Ss}$ in this age group (e.g., "hatchet," with a normative frequency of 8), and the third list (Lo-F/Lo-M) consisted of low-frequency concrete nouns (also less than 25 occurrences per million) whose meanings were unknown by the Ss (e.g., "dory," with a normative frequency of 7).

determined in the Ghatala and Levin (1974) study by having sixth-grade children pronounce and then define the words. (Any definition was taken to indicate that the word had meaning for the S.) The Lo-F/Hi-M words were those which at least $80 \%$ of the Ss could pronounce and define. The Lo-F/Lo-M words were those which at least $80 \%$ of the Ss could pronounce but no more than $20 \%$ could define. The mean Thorndike-Lorge frequency of the words selected for the Lo-F/Hi-M list was 5.93 and that for the Lo-F/Lo-M words was 5.80 .

All lists consisted of 15 pairs. Two versions of each list contained different random pairings of items. For each version of each list, one member of each pair was selected as correct; in a second list, the other members of the pairs were correct. Of the $16 \mathrm{Ss}$ in each condition, 4 were assigned to each list variation. All lists were constructed such that (a) the 15 pairs occurred in three random serial orders; (b) within any order, the correct members of the pairs occurred approximately equally often in the right and left positions; and (c) across orders, the correct member of a pair occurred no more than twice in the same position. The items in the pairs were typed side by side on $5 \times 8$ in. plain white cards and placed in notebooks. The correct members of the pairs were starred.

Procedure. The Ss were tested individually in a private room in the school building. Each $S$ received one silent (no-guess) anticipation trial followed by four anticipation response trials. The pairs were presented at a 5 -sec rate, timed by means of an electronic metronome. The $\mathrm{E}$ pronounced both words in a pair during the anticipation phase on all trials, and Ss indicated their choices by pointing.

\section{Results and Discussion}

Performance on the discrimination learning task is summarized in the last row of Table 1 . While the three means are in the predicted order, Dunnett tests $(\alpha=.05)$ revealed that, relative to $\mathrm{Hi}-\mathrm{F}, \mathrm{Lo}-\mathrm{F} / \mathrm{Lo}-\mathrm{M}$ was significantly inferior but Lo-F/Hi-M was not significantly superior. 
In order to analyze this result more fully, the previously described definitions test was administered to $\mathrm{Ss}$ in the Lo-F/Hi-M condition. As in the preliminary study, it was anticipated that Ss who would do well on the definitions test were those who also did well on the discrimination learning task. However, it is possible that this could be due simply to the fact that, in general, "brighter" Ss (i.e., Ss who know more low-frequency word definitions) are also better learners. In order to choose between a general hypothesis (that brighter children learn faster) and a specific hypothesis (that children who know the meanings of low-frequency items learn those particular items faster), Ss in the Hi-F and Lo-F/Lo-M conditions were also administered the Lo-F/Hi-M definitions test. To be consistent with the procedure followed in the discrimination task, E pronounced all of the words and, after hearing each word, $\mathrm{S}$ was required to define it.

The results are presented in Table 1 . The $48 \mathrm{Ss}$ were divided into two approximately equal-sized groups based on their definitions test performance. It may be seen that only in the Lo-F/Hi-M condition is there a substantial effect of knowing the definitions on discrimination performance. Looked at another way, for those Ss who knew most of the definitions (high: 27 or more correct out of 30 ), a nested comparison revealed that Lo-F/Hi-M Ss were superior to Hi-F Ss $(t=2.03$, $\mathrm{df}=42, \mathrm{p}<.05)$; but for those who knew fewer definitions (low: between 17 and 26 correct out of 30 ), no significant difference was observed $(|t|<1)$.

Thus, consideration of only the overall discrimination performance did not yield significant differences between Ss in the Lo-F/Hi-M and Hi-F conditions, contrary to predictions. Despite the procedural change suggested by the preliminary study, there was still appreciable variation among $S s$ in their knowledge of the meanings of the Lo-F/Hi-M words. However, the analysis of discrimination learning scores for the $\mathrm{Hi}-\mathrm{F}$ and Lo-F/Hi-M Ss as a function of their scores on the definitions test clearly supported the hypothesis that low-frequency words are better discriminated than high-frequency words, as long as Ss know the meanings of the low-frequency words.

The results of Experiment I provide some support for the notion that background frequency influences the difficulty of discrimination learning but that the meaningfulness of the stimuli is a variable moderating the effects of frequency. The data from this experiment were less than satisfying, however, because complete control over meaningfulness was not obtained with the materials used. Consequently, in the remaining experiments new high- and low-frequency materials were selected so that better control over meaningfulness could be obtained.

\section{EXPERIMENTS II AND III}

In Experiment II discrimination learning with revised Hi-F and Lo-F/Hi-M verbal materials was compared. As
Table 1

Mean Number of Correct Responses Over Four Test Trials by Experimental Condition and Performance on the Definitions Test (Experiment 1)

\begin{tabular}{cccc}
\hline & Hi-F & $\begin{array}{c}\text { Lo-F/ } \\
\text { Hi-M }\end{array}$ & $\begin{array}{c}\text { Lo-F/ } \\
\text { Lo-M }\end{array}$ \\
\hline \multirow{3}{*}{ High Definitions } & 47.75 & 53.22 & 40.33 \\
& $(\mathrm{~N}=8)$ & $(\mathrm{N}=9)$ & $(\mathrm{N}=6)$ \\
Low Definitions & 47.12 & 45.00 & 41.90 \\
& $(\mathrm{~N}=8)$ & $(\mathrm{N}=7)$ & $(\mathrm{N}=10)$ \\
Weighted Means & 47.44 & 49.62 & 41.31 \\
\hline
\end{tabular}

Note-MS $=30.62$

in the previous experiment, it was expected that the Lo-F/Hi-M materials would produce superior learning. However, in order to demonstrate that this finding could not be attributable simply to the particular idiosyncracies of the materials selected, in Experiment III the same materials were compared under free-recall learning conditions. Based on the well known differential effects of word frequency on recognition. and recall-type measures of learning (cf. Kintsch, 1970), it was expected that the present Lo-F/Hi-M materials, while superior to Hi-F materials in discrimination learning, would be inferior in free recall.

In addition, experimental line drawings corresponding to the verbal stimuli were included in order to see if these would be similarly responsive to background frequency manipulations. Although unforeseen at the time, the inclusion of pictorial materials provided data relevant to current theorizing about picture-word differences in learning (cf. Paivio, 1971). Such issues will be discussed following a presentation of the experimental results.

\section{Method}

Materials. Fifty-two concrete nouns were selected, with half designated as $\mathrm{Hi}-\mathrm{F}$ and half as Lo-F as determined from Carroll, Davies, and Richman's (1971) word frequency norms (third-grade level). By selecting from actual materials used by children, we sought to obtain more realistically based high- and low-frequency words than those determined from more remote norms such as those of Thorndike and Lorge (1944). In particular, the Carroll et al norms are derived from samples of children's reading materials, grade level by grade level. Overall, the Lo-F words (mean of 7.5 occurrences in third-grade materials, range of 1 to 19) appeared in such samples much less frequently than the Hi-F words (mean of 351.5 , range of 232 to 785). An attempt was made to match Hi-F and Lo-F words with respect to their general object class (e.g., "dog" with "ape," "window" with "chimney") as much as was possible. Line drawings of each of these items were also created.

The final selection of words and pictures resulted from initial pilot testings with a larger sample of materials. In the pilot tests, there were two major concerns: (1) that the Hi-F and Lo-F items generated from the Carroll et al (1971) norms corresponded to our Ss' phenomenal experience with such items and (2) that Ss possessed the desired labels for each of the pictures (thereby also indicating that all materials were "meaningful" to Ss). To deal with the first concern, we presented mixed-frequency word pairs aurally to beginning fourth graders, with Ss instructed to circle the letter on supplied answer sheets which corresponded to the word in each pair that they had "heard, seen, or used more 
often." To deal with the second concern, we showed the pictures one at a time to additional Ss and asked them to label each one. Following these procedures, it was possible to select $26 \mathrm{HiF}$ and $26 \mathrm{Lo}-\mathrm{F}$ items which met at least an $80 \%$ agreement criterion on both the phenomenal frequency judgment and the picture labeling tasks. From these items, $13 \mathrm{Hi}-\mathrm{F}$ pairs were randomly formed for the discrimination learning task (Experiment II), with one item in each pair designated correct. The matched (from the pilot studies) Lo-F items were then selected to form comparable pairs. A random sample of 18 of the Hi-F items and their Lo-F counterparts were selected for the free-recall task (Experiment III). Both verbal and pictorial items appeared on cards inserted into looseleaf binders.

Subjects and Design. A total of 123 fourth graders (different from those used in the pilot studies) from an elementary school in the Midwest participated in the two experiments. Of these, 75 Ss participated in Experiment II and 48 in Experiment III. Within each experiment, Ss were randomly assigned in approximately equal numbers to the four cells of the design as defined by the combination of frequency (Hi-F vs Lo-F) and modes (words vs pictures).

Procedure. In the discrimination task, Ss were shown each paix for $3 \mathrm{sec}$ under the anticipation method (with one silent study trial). Pilot tests with the new materials revealed that a 3 -sec rate was appropriate. Additionally, E pronounced the two pair members during the anticipation phase in the word conditions only (to guarantee that each word would be recognized). Following the initial study trial, two response trials were provided.

In the free-recall task, stimuli were presented one at a time for $3 \mathrm{sec}$ apiece. As in the discrimination task, E named aloud each stimulus in the word conditions during presentation. Four presentation trials, each followed by a S-paced recall trial, were provided.

\section{Results and Discussion}

Mean performance (expressed as percentages) on the two tasks is presented in Fig. 1. On the free-recall task, S-produced labels that were synonymous with the intended ones were scored as correct in the picture conditions. (The mean square error associated with total correct responses on the two-trial 13-pair discrimination task was 10.18 and that with total correct responses on the four-trial 18-item free-recall task was 51.36.)

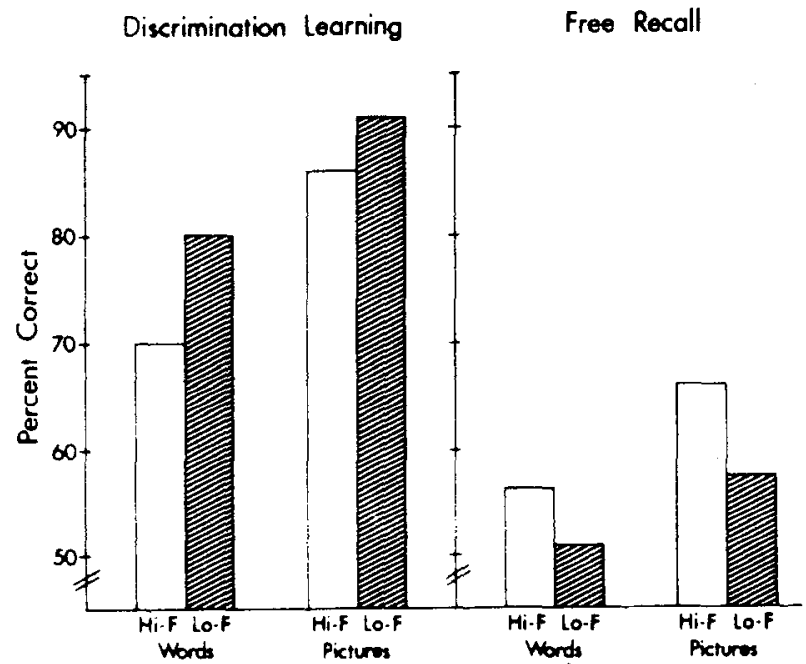

Fig. 1. Mean percent correct responses by experimental condition in discrimination learning (Experiment II) and free recall (Experiment III).
Since there were slightly unequal numbers of Ss in the four discrimination learning conditions, the harmonic mean was computed $(\widetilde{\mathrm{n}}=18.72)$ and unweighted means analysis of variance applied to the two factors. According to this procedure, pictures were discriminated better than words $(F=20.26$, df $=1 / 71, p<.001)$, a result in accord with previous data for both children (e.g., Wilder \& Levin, 1973) and adults (e.g., Rowe \& Paivio, 1971a). Moreover, as anticipated with meaningful materiais, Lo-F was found to be superior to $\mathrm{Hi} \cdot \mathrm{F}$ $(\mathrm{F}=7.36, \mathrm{df}=1 / 71, \mathrm{p}<.01)$. The Frequency by Modes interaction was not significant $(\mathrm{F}<1)$.

The picture-over-word effect remained on the free-recall task $(F=10.76, d f=1 / 44, p<.01)$, which is consistent with previous results (e.g., Cole, Frankel, \& Sharp, 1971; Paivio \& Csapo, 1969). However, as was expected, the frequency effect reversed, that is, Hi-F stimuli were better recalled than Lo-F stimuli $(F=15.68, d f=1 / 44, p<.001)$. Once again, the interaction was nonsignificant $(F=1.93, d f=1 / 44$, $\mathrm{p}>.10)$.

Thus, the major premise of the present research (viz., that background frequency is negatively related to discrimination learning performance) was supported. Conversely, a positive relationship between background frequency and free-recall performance was observed, suggesting that different cognitive processes were evoked by the two tasks even though they included the same materials.

Since no interaction between frequency and stimulus mode was obtained in either task, it might be concluded that background frequency influences the learning of pictorial as well as verbal materials. While this seems obvious in the case of free recall (where $S$ must store and retrieve the labels of stimuli that are identical for both types of material), it is less so in the case of discrimination learning (where production of verbal labels of the stimuli is not required). However, recent evidence (cf. Tversky, 1973) suggests that verbal processes are involved in ostensibly nonverbal tasks (such as the pictorial discrimination task of the present study). Consequently, additional data to those of Experiment II were collected to corroborate the existence of the effect with pictures. Seventeen fourth graders from the same school used in Experiment II were given a much longer (26-pair) list, formed by including both the previous Hi-F and Lo-F picture pairs in a single mixed list. The obtained difference of 1.65 correct responses in favor of Lo-F was statistically significant $(\mathrm{t}=2.04, \mathrm{df}=16, \mathrm{p}<.01)$, with $11 \mathrm{Ss}$ performing better on Lo-F pairs and only 3 Ss performing better on $\mathrm{Hi}-\mathrm{F}$ pairs (and 3 Ss showing no difference).

Two recent accounts of the picture-word differences found in a variety of learning tasks have been offered by Paivio (1971). The "concreteness" explanation asserts that visual imagery is a powerful determinant of learning efficiency and that pictures elicit such imagery more directly than do their associated verbal labels. The "dual-coding" explanation, on the other hand, asserts 
that two internal codes (imaginal and verbal) are more efficient than one and that pictures are more likely to produce such a dual coding than words-due to the presumed greater propensity of $S$ s to label pictures spontaneously than to generate visual images for words spontaneously.

Paivio and Csapo (1973) have provided evidence in support of the "dual-coding" (rather than the "concreteness") interpretation of picture-word differences in free recall. While this also seems to be the case in discrimination learning (Levin, Ghatala, DeRose, Wilder, \& Norton, in press), the present study suggests that neither explanation is completely adequate if "background frequency" is ignored. That is, neither can satisfactorily account for discrimination learning differences between high- and low-frequency materials of comparable concreteness (i.e., between high- and low-frequency words or between high- and low-frequency pictures). However, in light of the present finding that pictures with low-frequency labels were better discriminated than were those with high-frequency labels, Paivio's suggestion that pictures are dually encoded (i.e., verbally, along with their nonverbal images) is eminently reasonable in its own right and should be considered in conjunction with the hypothesis that picture-word differences in discrimination tasks may also be due to the differing background frequencies of the two types of material (cf. Ghatala et al, 1973).

At the same time, even though a "background frequency" effect was produced with pictorial materials here, its locus is difficult to trace. That is, are low-frequency pictures discriminated better than high-frequency pictures because their respective verbal labels differ in background frequency or because their respective object referents differ in background frequency, or both? Certainly the word "giraffe" is encountered less frequently by (North American) Ss than is the word "cat;" yet Ss also encounter more cats than giraffes in their day-to-day existence. Although the present research does not lend itself to a teasing apart of these naturally correlated factors, it would seem possible to do so.

\section{GENERAL DISCUSSION}

The present series of experiments indicates that background frequency influences the discrimination learning of verbal materials in a manner prescribed by Weber's law. More importantly, however, the experiments have shown that the negative relationship between frequency and discrimination learning is evident only for verbal materials which are meaningful to Ss. The analysis in Experiment $I$, which showed that only those Ss in the Lo-F/Hi-M group who actually knew the definitions of the Lo-F/Hi-M words performed significantly better than comparable $\mathrm{Ss}$ in the $\mathrm{Hi}-\mathrm{F}$ group, appears to be particularly strong evidence in support of the frequency/meaningfulness hypothesis advanced by Ghatala and Levin (1974).

The first experiment revealed substantial individual differences among children in their knowledge of word meanings, which in turn illustrates the importance of selecting high- and low-frequency words that are not contaminated by differences in meaningfulness. While such individual differences in word knowledge might be expected to be reduced with adult $\mathrm{Ss}$, it is not unlikely that they still exist (particularly with very low-frequenty words) and may be responsible for the equivocal findings concerning frequency effects in verbal discrimination learning which were described earlier. In this regard, Experiment II demonstrated that, when words are chosen which are uniformly high in meaningfulness for all Ss, then the negative relationship between frequency and discrimination learning is clearly apparent.

Experiments II and III substantiated the finding of opposite effects of word frequency in free recall and discrimination learning (the latter presumably involving recognition memory). Such differential effects of frequency, as well as other variables, have led some (e.g., Kintsch, 1970; Underwood, 1969) to propose that different processes or memory attributes underlie recognition and recall.

Finally, background frequency appeared to influence the discrimination learning of pictures as well as words, although, as noted earlier, further research is needed to determine the locus of the frequency effect. Furthermore, in the present study the object referents of all pictures were mearingful to the Ss. It would be of interest to determine if the discrimination learning of pictures is also moderated by meaningfulness, as has been demonstrated here for verbal materials.

\section{REFERENCES}

Carroll, J. B., Davies, P., \& Richman, B. The American heritage work frequency book. New York: Houghton Mifflin, 1971.

Cole, M., Frankel, F., \& Sharp, D. Development of free recal learning in children. Developmental Psychology, 1971, 4, 109-123.

Ekstrand, B. R., Wallace, W. P., \& Underwood, B. J. A frequency theory of verbal-discrimination learning. Psychological Review, 1966, 73, 566-578.

Ghatala, E. S.. \& Levin, J. R. Developmental differences in frequency judgments of words and pictures. Journal of Experimental Child Psychology, 1973, 16, 495-507.

Ghatala, E. S., \& Levin, J. R. Discrimination learning as a function of differences in materials: A proposed explanation. Memory \& Cognition, 1974, 2, 395-400.

Ghatala, E. S., Levin, J. R., \& Wilder, L. Apparent frequency of words and pictures as a function of pronunciation and imagery. Journal of Verbal Learning \& Verbal Behavior, 1973, $12,85-90$.

Ingison, L. J., \& Ekstrand, B. R. Effects of study time, method of presentation, word frequency, and word abstractness on verbal discrimination learning. Journal of Experimental Psychology, 1970, 85, 249-254.

Kintsch, W. Models for free recall and recognition. In D. A. Norman (Ed.), Models of human memory. New York: Academic Press, 1970.

Levin, J. R., Ghatala, E. S., \& DeRose, T, M. The effect of stimulus prefamiliarization on children's discrimination 
learning. Technical Report No. 285. Madison: Wisconsin Research \& Development Center for Cognitive Learning, in press.

Levin, J. R., Ghatala, E. S., DeRose, T. M., Wilder, L., \& Norton, R. W. A further comparison of imagery and vocalization strategies in children's discrimination learning. Journal of Educational Psychology, in press.

Paivio, A. Imagery and verbal processes. New York: Holt, 1971.

Paivio, A., \& Csapo, K. Concrete-image and verbal mem ory codes. Journal of Experimental Psychology, 1969, 80, 279-285.

Paivio, A., \& Csapo, K. Picture superiority in free recall: Imagery or dual coding? Cognitive Psychology, 1973, 5, 176-206.

Paivio, A., \& Rowe, E. J. Noun imagery, frequency, and meaningfulness in verbal discrimination. Journal of Experimental Psychology, 1970, 85, 264-269.

Paivio, A., Y uHe, J. C., \& Madigan, S. Concreteness, imagery and meaningfulness values for 925 nouns. Journal of Experimental Psychology Monograph Supplement, 1968, $76(1$, Part 2).

Rowe, E. J., \& Paivio, A. Discrimination learning of pictures and words. Psychonomic Science, 1971a, 22, 87-88.

Rowe, E. J., \& Paivio, A. Word frequency and imagery effects in verbal discrimination learning. Journal of Experimental Psychology, 1971b, 88, 319-326.

Thorndike, E. L., \& Lorge, I. The teacher's word book of 30,000 words. New York: Bureau of Publications, Teachers College, Columbia University, 1944.
Tversky, B. Encoding processes in recognition and recall. Cognitive Psychology, 1973, 5, 275-287.

Underwood, B. J. Attributes of memory. Psychological Review, $1969,76,559-573$

Underwood, B. J., Broder, P. K., \& Zimmerman, J. Retention of verbal discrimination lists as a function of number of prior lists, word frequency, and type of list. Journal of Experimental Psychology, 1973, 100, 101-105.

Underw ood, B. J., \& Schulz, R. W. Meaningfulness and verba learning. Philadelphia: Lippincott, 1960.

Wilder, L., \& Levin, J. R. A developmental study of pronouncing responses in the discrimination learning of words and pictures. Journal of Experimental Child Psychology, 1973, 15, 278-286.

(Received for publication February 25, 1974; revision received April 26, 1974.)

(Accepted for publication May 3, 1974.) 